\title{
Development of EEG Amplifier and Derivation of BIS Algorithm
}

\author{
Jyoti Pande ${ }^{1}$, Hiranya Kudva ${ }^{2}$, Prof. Jyothi Warrier ${ }^{3}$ \\ I(Biomedical Department,MGM College of Engineering and Technology, Mumbai University,India) \\ ${ }^{2}$ (Biomedical Department,MGM College of Engineering and Technology, Mumbai University,India) \\ ${ }^{3}$ (Biomedical Department,MGM College of Engineering and Technology, Mumbai University,India)
}

\begin{abstract}
The raw EEG signals are captured from the brain, specifically Delta waves. The signal is amplified, rectified and processed mathematically. Using FFT EEG parameters are drawn which are further processed with complex algorithm to result in BIS score ranging from 0 to 100. Using this score the depth of anesthesia is calculated.
\end{abstract}

Keywords : BIS; EEG; anesthesia; bispectral analysis; hypnosis.

\section{INTRODUCTION}

The bispectral index (BIS) is a fairly recent development used to measure the effects of anesthetics and sedatives on the brain and consciousness. It uses a complex mathematical algorithm based upon descriptive EEG parameters from the frontal cortex to suggest various levels of sedation. A BIS index is derived ranging from 0 to 100. Here 0 denotes maximum effect of anesthesia and 100 denotes awake state. Basically in BIS delta waves are considered which are captured from frontal part of the brain. Delta waves are captured because it is associated with the deep sleep.

\section{THE ELECTROENCEPHALOGRAM}

The traditional EEG is defined as a time domain recording of spontaneous electrical signals formed by the summation of the activity of millions of neurons in the cerebral cortex. The activity is produced from excitatory and inhibitory post-synaptic potentials in large pyramidal neurons located in the lower layers (e.g. layer $\mathrm{V}$ ) of the cerebral cortex. Measures of the electrical signal such as amplitude and frequency are modulated by afferent inputs transmitted from sensory-specific thalamic nuclei serving as gates between receptors and the cortex and by 'epicentres' within cortical areas [1].

\section{Resting Rhythms of the Brain}

EEGs show continuous oscillating electric activity. The amplitude and the patterns are determined by the overall excitation of the brain which in turn depends on the activity of the reticular activating system in the brain stem. Amplitudes on the surface of the brain can be up to $10 \mathrm{mV}$, those on the surface of the scalp range up to $100 \mathrm{mV}$. Frequencies range from 0.5 to $100 \mathrm{~Hz}$. The pattern changes markedly between states of sleep and wakefulness.

Four classes of wave groups are described: alpha, beta, theta and delta.

Alpha

Beta

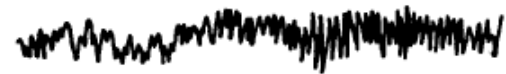

Theta

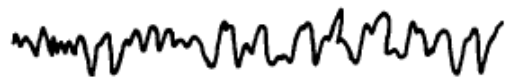

Delta

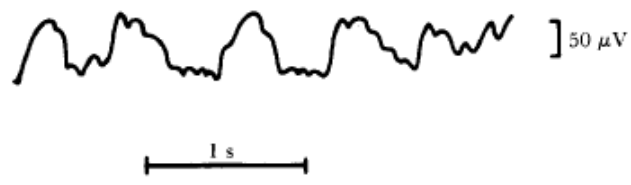

Fig. 1. Normal EEG patterns 
Alpha waves: Alpha waves contain frequencies between 8 and $13 \mathrm{~Hz}$ with amplitude 2 to $200 \mu \mathrm{V}$. They are found in normal people who are awake and resting quietly, not being engaged in intense mental activity.

Beta waves: Beta waves have a frequency range of 14 to $22 \mathrm{~Hz}$, extending to $50 \mathrm{~Hz}$ under intense mental activity.

Theta waves: Theta waves have a frequency range between 4 to $7 \mathrm{~Hz}$ with an amplitude of $10 \mu \mathrm{V}$. Sudden removal of something causing pleasure will cause about $20 \mathrm{~s}$ of theta waves.

Deta waves: Delta waves have a frequency content between 0.5 and $4 \mathrm{~Hz}$ with an amplitude 20 to $200 \mu \mathrm{V}$. They occur during deep sleep, during infancy and in serious organic brain disease.

\section{Electrode Configuration}

In process of BIS calculation, the first step is acquisition of EEG signal, which is made through application of four electrodes placed on the skin surface that enable an appropriate electrical conduction with low impedance. Electrodes are placed in each of the following four positions: The left and right temporal region, between the lateral edge of the eye and the upper edge of the ear; the middle of the frontal region (referential electrodes); and lateral to the referential electrode (ground electrodes).The assembly used is the unilateral referential with exploratory electrode in FT9 position (frontal-temporal region) and reference electrode in the FPz position (front polar) [2]. This determines that the obtained EEG lineation is monocanal (left or right, according to position of frontal-temporal electrode). Electrode in the AF7 position is used in BIS algorithm to increase its calculation in the presence of electromyographic activity, and the FP1 electrode (virtual ground) has the purpose of increasing the rejection of common mode [3].

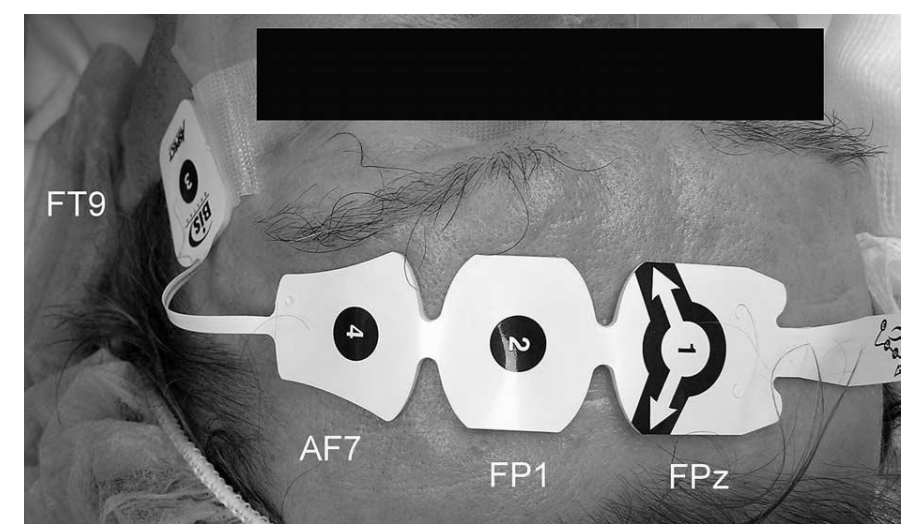

Fig. 2. Placement of electrodes for BIS

\section{THE BIS INDEX}

The BIS Index is a processed EEG parameter with extensive validation and demonstrated clinical utility. It is derived utilizing a composite of measures from EEG signal processing techniques including bispectral analysis, power spectral analysis, and time domain analysis. These measures were combined via an algorithm to optimize the correlation between the EEG and the clinical effects of anesthesia, and quantified using the BIS Index range. Bispectral analysis is a signal processing methodology that assesses relationships among signal components and captures synchronization within signals like the EEG. By quantifying the correlation between all the frequencies within the signal, bispectral analysis yields an additional EEG facet of brain activity. 


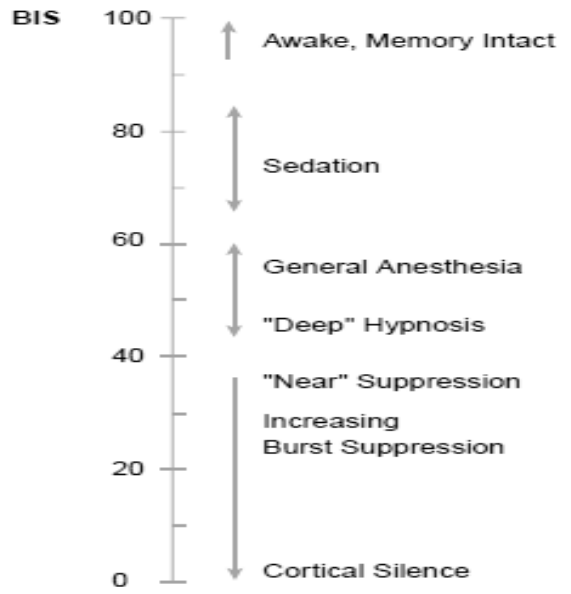

Fig. 3. BIS Index Range

IV. BLOCK DIAGRAM

To derive the BIS index the first stage is acquisition of EEG from patient. This is done by the 4electrode system as explained previously. The acquired signal goes through many stages to finally give the BIS index. The following diagram shows the various blocks for converting Raw EEG signal to desired BIS index.

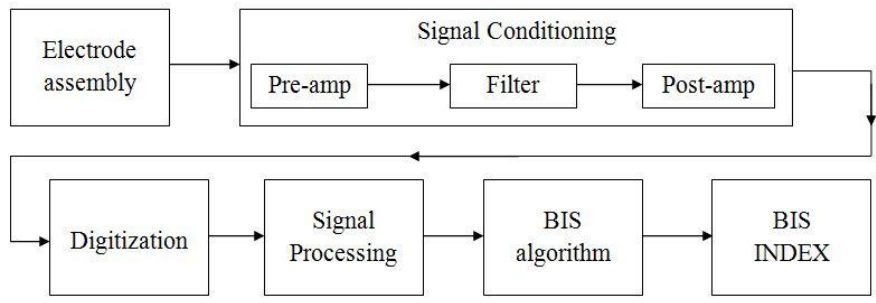

Fig. 4. Block Diagram

\section{a. Electrode Assembly}

In process of BIS calculation, the first step is acquisition of EEG signal, which is made through application of four electrodes placed on the skin surface that enable an appropriate electrical conduction with low impedance. After acquisition the signal undergoes amplification and filtering.

\section{b. Pre-amplifier}

One of the key features of the above block diagram level design is that the input and output signals are capacitively coupled, while the internal stages of the preamplifier are DC coupled. This capacitive coupling provides both rejection of low frequency signals that would signal, as well as providing a block for any DC currents that might flow through the patient's electrodes. Capacitive coupling eliminates any DC micro shock hazards, and helps greatly to increase the safety of the overall system. Instrumentation amplifier is to be used as pre-amplifier with high CMRR and adjustable gain.

\section{c. Filter}

EEG signal frequency recorded generally ranges from 0.5 to $100 \mathrm{~Hz}$. All the added noise is normally filtered at this stage. Band pass and Notch filter are used for removing noise and baseline interference. A lowfrequency filter minimizes low frequency baseline oscillations that are not of biological origin but should affect the low frequency EEG as little as possible. Some systems strongly filter the low-frequency EEG so that the recorded signal appears noise-free.

\section{d. Post-amplifier}

To amplify the signal further to make it suitable for output to some recording device. This is the final amplifying stage with high gain.

\section{e. Digitization}

Digitalization is performed after acquisition and amplification of signal. The captured analog signal is presented in regular intervals (frequency expressed in $\mathrm{Hz}$ ) so that deflections of each wave are defined by a series of positive or negative concrete values dependent of the moment of data collection. The frequency of 
collected data is essential for obtaining a safe digitalized signal as, according to Shannon's theorem, it must be superior to double of maximum frequency of the analyzed signal. Maximum frequencies of EEG signal have been considered for a long time, from 30 to $40 \mathrm{~Hz}$, therefore, $70 \mathrm{~Hz}$ of frequency would be more real. If the frequency of samples is small, there is a risk of erroneously converting, a fast analog wave into a slow digitalized wave (aliasing effect).

\section{f. Signal processing}

After digitalization, the signal undergoes a process of artifacts recognition. The artifacts produced by signals that exceeded dynamic limit of amplifier may be identified in epoch and then are rejected, since original data cannot be reconstituted. Other artifacts can be eliminated from contaminated signal and resulting filtered signal may be used for further analysis.

\section{g. BIS algorithm}

The BIS algorithm is the algorithm which gives the final BIS index. It includes bispectral analysis, power spectral analysis and time domain analysis of the digitized EEG signal. The BIS number is obtained from weighted analysis of four sub parameters: burst suppression ratio, QUAZI suppression, beta relative power and fast/slow synchronization which are explained in the next chapter.

\section{MATHEMATICAL CONCEPT OF BIS}

EEG signal after digitalization and filtration of artifacts can be mathematically treated. However, at this moment alterations in voltage can only be evaluated in time domain. From these parameters (voltage and time), many statistical analyses can be carried out resulting in important variables. For statistical analysis of these data in time domain it is necessary to know that EEG is a non deterministic signal, in other words, it is not possible to exactly predict its future values. Therefore, EEG is a stochastic signal and some statistical points are not predictable. In BIS calculation, its generation is based on two measurements of EEG waves: burst suppression ratio and QUAZI suppression index.

\section{a. Burst Suppression Ratio (BSR)}

The BSR is a time-domain EEG parameter that was developed to measure burst suppression. During deep anesthesia, the EEG may develop a peculiar pattern of activity characterized by alternating episodes of normal-to high- voltage activity changing to low voltage or even isoelectricity, thereby rendering the EEG inactive in appearance. This pattern of EEG is called burst suppression [4]. Suppression rate is defined as intervals over 0.5 seconds in which EEG voltage is below $\pm 0.5 \mu \mathrm{V}$. Suppression rate is the epoch fraction (time period of the analysis of two seconds) in which EEG is isoelectric (does not exceed $\pm 0.5 \mu \mathrm{V}$ ). Due to the especially variable nature of suppression rate, it must be calculated on average during an interval of at least 30 epochs (60 seconds). Regular suppression ratio is zero.

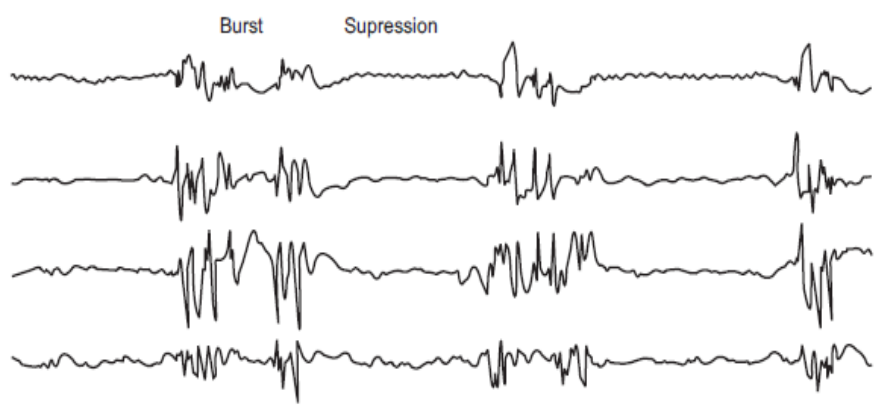

Fig.5. Presence of burst-suppression

\section{b. QUAZI Suppression Rate}

QUAZI suppression rate was projected to discover the presence of suppression rates in the presence of erratic voltage of baseline. QUAZI incorporates information of slow waves $(<1.0 \mathrm{~Hz})$, derived from frequency domain to detect the activity of superimposed rates over these slow waves that would somehow contaminate original algorithm of burst suppression ratio (BSR), exceeding voltage criteria established to define electrical silence. With this index, we can detect certain suppression periods that could not be discovered with strict criteria of electrical silence $( \pm 0.5 \mu \mathrm{V})$ imposed by definition of burst suppression rate. 


\section{c. BetaRatio}

In clinical monitors, EEG is decomposed into its frequency spectrum by Fast Fourier Transform (FFT). This algorithm enables an efficient calculation of digitalized data and is presented graphically as a histogram of power in frequency domain, being discarded the phase spectrum. Quantitative analysis of signal obtained through the FFT enables the identification of some general patterns, called bands, where each is defined by a range of frequencies:

$\delta=0.5-3.5 \mathrm{~Hz}, \theta=3.5-7.0 \mathrm{~Hz}, \alpha=7.0-13.0 \mathrm{~Hz}, \beta=13.0-30.0 \mathrm{~Hz}$ and $\beta 2=30.0-50.0 \mathrm{~Hz}$.

The frequency analysis parameter that uses BIS is the $\beta$ relative rate also known as BetaRatio, which is defined as $\log \left(\mathrm{P}_{30-47} \mathrm{~Hz} / \mathrm{P}_{11-20} \mathrm{~Hz}\right)$. In other words, it is the logarithm of the quotient between sums of spectral energies (wave amplitude expressed as square voltage) of frequencies bands. Thus, we have a low-frequency band $(11-20 \mathrm{~Hz})$, which is included within two classic spectra: $\alpha$ and $\beta$ and another one of high frequency, included within $\beta 2$ spectrum. BIS analysis weights the BetaRatio most heavily when the EEG has the characteristics of light sedation.

d. Bispectral Analysis

Bispectral analysis incorporates information about the phase related to beginning of considered epoch, from different frequencies obtained. Bispectrum measures phase correlation of waves obtained by Fourier analysis among different frequencies. Bispectral analysis enables to suppress noise Gaussian sources, increasing relationship signal/noise, being able to identify non linear situations important in process of signal generation. Bispectrum is calculated multiplying three complex spectral values (each complex spectral value includes frequency, amplitude and phase information), the spectral value of $\mathrm{f} 1$ and $\mathrm{f} 2$ primary frequencies by spectral value of modulation frequency $(\mathrm{f} 1+\mathrm{f} 2)$. This product is the most important point of bispectral analysis: if in each frequency of tripod ( $\mathrm{f} 1, \mathrm{f} 2$ and $\mathrm{f} 1+\mathrm{f} 2$ ) spectral amplitude is big (there is some sine waive for this frequency) and if phase angles for each of three considered frequencies are aligned, the final product will be big. On the contrary, if one of sine components is small or absent, or if phase angles are not aligned, the product will be small [5]. Bispectrum is expressed in microvolt raised to the third power $(\mu \mathrm{V} 3)$ as it is product of three sine waves, each one with amplitude in micro volts. A value derived from bispectrum is bicoherence, which numerically varies from 0 to 1 proportionally to degree of phase coupling in frequency of considered tripod.

\section{e. SyncFastSlow}

BIS uses as parameter derived from bispectral analysis fast/ slow synchronization, which is logarithm of quotient between sum of all bispectrum peaks in band from 0.5 to $47 \mathrm{~Hz}$ and sum of bispetrum in band from 40 to $47 \mathrm{~Hz}$. The SyncFastSlow parameter correlates with behavioral responses during moderate sedation or light anesthesia. SynchFastSlow predominates during EEG activation (the excitement phase) and during surgical levels of hypnosis.

\section{VI. generation of bis}

BIS number is obtained from weighted analysis of four sub parameters: burst suppression ratio, QUAZI suppression, beta relative power and fast/slow synchronization, where it is applied a statistical multivariate model using a non linear function. The particular utilization of many sub parameters in BIS generation was empirically derived from a database, prospectively accumulated, of EEG and sedation scales in which it was used a great variety of anesthetic protocols. Each of these sub parameters presents greater or smaller influence in BIS generation, depending on variations in electrical activity captured by the explorer electrode. 


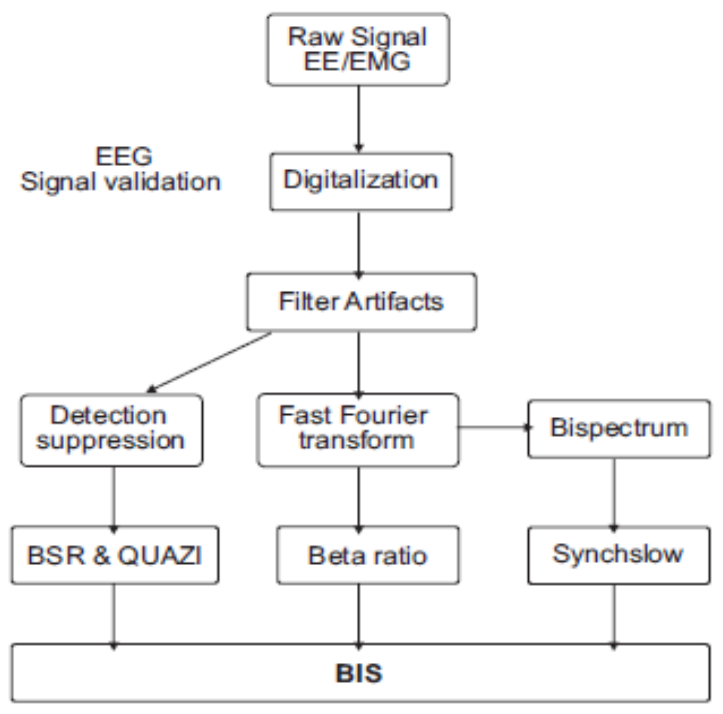

Fig.6. Sub parameters generating BIS

So, we have:

- Fast/slow synchronization - correlates better with answers during moderate sedation or superficial anesthesia. This parameters also correlates well with EEG activation states (excitation phase) and during surgical levels of hypnosis.

- BetaRatio - this parameter is the most important for calculation algorithm of BIS during superficial sedation.

- Burst suppression and QUAZI suppression - detect deep anesthesia.

\section{ACKNOWLEDGEMENT}

We WOULD LIKE TO THANK OUR HEAD OF THE DEPARTMENT DR. G.D. JINDAL FOR GIVING US THE FREEDOM TO SELECT OUR DESIRED TOPIC FOR THE PROJECT WORK AND SUPPORTING US IN OUR ENDEAVORS.

\section{REFERENCES}

[1] Klaus A Otto, "EEG power spectrum analysis for monitoring depth of anaesthesia during experimental surgery," Lab Anim January 2008 Vol. 42, pp. 45-61.

[2] Jay W. Johansen, "Update on Bispectral Index monitoring,” Best Practice \& Research Clinical Anaesthesiology, Vol. 20, No. 1, 2006, pp. 81-99.

[3] Rogean Rodrigues Nunes et al, "Bispectral Index and Other Processed Parameters of Electroencephalogram: an Update," Revista Brasileira de Anestesiologia 105, Vol. 62, No. 1, January-February, 2012.

[4] Bispectral Index Monitor An evidence based analysis, Ontario Health Technology Assessment Series 2004; Vol. 4, No. 9.

[5] Proakis JG, Rader CM, Ling F and Nikias CL - "Signal analysis with higher order spectra," Advanced Digital Signal Processing, ${ }^{\text {a }}$ Edition, New York, Macmillan, 1992, pp. 550-89. 